\title{
Detection of mRNA by in situ hybridisation and in northern blot analysis using oligodeoxynucleotide probes labelled with alkaline phosphatase
}

\author{
M A Farquharson, R Harvie, A Kennedy, A M McNicol
}

\begin{abstract}
Aims: To assess whether a reduction in intensity of signal observed using an alkaline phosphatase labelled oligodeoxynucleotide probe could be explained on the basis of procedural steps rather than reduced sensitivity.

Method: Signal intensity was assessed on in situ hybridisation for pro-opiomelanocortin (POMC) mRNA in rat pituitary and for somatostatin mRNA in human pancreas and in northern blot analysis for POMC mRNA in the presence and absence of formamide. The direct effects of formamide on the alkaline phosphatase detection step were also assessed using histochemical enzyme detection in rat kidney.

Results: All signals were reduced in systems containing formamide.

Conclusions: In the absence of formamide clear, strong signals for specific mRNAs can be obtained by in situ hybridisation and northern blot analysis using oligodeoxynucleotide probes directly labelled with alkaline phosphatase. Formamide seems to inhibit the activity of alkaline phosphatase.
\end{abstract}

(F Clin Pathol 1992;45:999-1002)

The development of non-isotopic in situ hybridisation techniques based on synthetic oligodeoxynucleotide probes has concentrated to date on 3' tailing with nucleotides coupled to biotin $^{1-3}$ or digoxigenin. ${ }^{45}$ An alternative approach is the direct linkage of a reporter molecule to the 5 ' base. Sites of hybridisation are then detected by a single-step histochemical reaction. In diagnostic practice this would have the advantage of reducing the time required for the technique by eliminating the intermediate binding steps of avidin to biotin or of antibody to digoxigenin. However, by limiting to one the number of reporter molecules on each probe, it might reduce the sensitivity to levels unacceptable for general use, even with high copy sequences. We therefore set out to compare the in situ hybridisation signal for pro-opiomelanocortin (POMC) messenger RNA (mRNA) in the rat pituitary gland, using the same probe sequence 3 ' end-labelled with digoxigenin ${ }^{4}$ or 5 ' linked to alkaline phosphatase. Initial results indicated that the signal obtained with the alkaline phosphatase linked probe was much weaker than that generated by the digoxigenin-labelled sequence. We therefore performed a series of experiments to determine whether this reflected a significant reduction in sensitivity or whether it could be explained on the basis of some other aspect of the hybridisation procedure.

\section{Methods}

Pituitary glands were removed from adult male Sprague-Dawley rats. These were fixed in $10 \%$ neutral buffered formalin and processed to paraffin wax or snap frozen in liquid nitrogen and stored at $-70^{\circ} \mathrm{C}$ for RNA extraction. Rat kidneys were snap frozen in liquid nitrogen and stored at $-70^{\circ} \mathrm{C}$. Surgical specimens of human pancreas were obtained from the files at the Glasgow Royal Infirmary. These had also been fixed in $10 \%$ neutral buffered formalin and processed to paraffin wax. Sections, $5 \mu \mathrm{m}$ thick, were mounted on slides coated with 3-aminopropyltriethoxysilane.

Oligodeoxynucleotide probes were synthesised with an amino-linked base at the $5^{\prime}$ end. These were:

(i) 5' d(CTTGCCCCAGCGGAAGTGCTCCAT) $3^{\prime}$

complementary to the sequence of POMC mRNA encoding the adrenocorticotrophin (ACTH) 4-11 sequence. ${ }^{6}$

(ii) 5' d(GTCTTCCAGAAGAAGTTCTTGCAGCC) 3',

complementary to the sequence of preprosomatostatin mRNA encoding residues $16-24$ of somatostatin $28 .^{7}$

These were conjugated to alkaline phosphatase using the E-LINK oligonucleotide labelling kit (Cambridge Research Biochemicals).

All prehybridisation buffers were treated with $0 \cdot 1 \%$ diethylpyrocarbonate (DEPC) (overnight then autoclaved) to destroy any ribonucleases.

(i) For in situ hybridisation and northern blot analysis, $50 \%$ formamide buffer, consisting of $10 \mathrm{mM}$ TRIS-HC1, $\mathrm{pH} 7 \cdot 5$, $12.5 \times$ Denhardt's solution, $2 \times$ SSC $(1 \times \mathrm{SSC}=0 \cdot 15 \mathrm{M}$ sodium chloride, $0.015 \mathrm{M}$ sodium citrate) $50 \%$ formamide, $0.5 \%$ sodium dodecyl sulphate (SDS), $10 \%$ dextran sulphate, $250 \mu \mathrm{g} / \mathrm{ml}$ salmon sperm DNA.

(ii) For in situ hybridisation, $0 \%$ formamide buffer, prepared as above, but replacing formamide with water.

(iii) For blots, $0 \%$ formamide buffer contained $5 \times$ SSC, $0.5 \%$ SDS, $5 \times$ Denhardt's solution. 
Hybridisation was performed as described before. ${ }^{4}$ Briefly, the sections were subjected to the following pretreatments before probe application: $0.2 \mathrm{M} \mathrm{HCl}$ for 20 minutes, $0.3 \%$ Triton X-100 (Sigma) for 15 minutes, digestion with $20-50 \mu \mathrm{g} / \mathrm{ml}$ proteinase $\mathrm{K}$ (Sigma) for 30 minutes at $37^{\circ} \mathrm{C}, 0.2 \%$ glycine for one minute and finally post-fixed in $4 \%$ paraformaldehyde. The sections were washed in phosphate buffered saline (PBS) between pretreatments. The probe was applied in either $0 \%$ or $50 \%$ formamide hybridisation buffer.

Slides of rat pituitary were incubated with the POMC probe at $42^{\circ} \mathrm{C}$ overnight and those of human pancreas with the somatostatin probe at $37^{\circ} \mathrm{C}$ overnight. The post-hybridisation washes consisted of one wash in $4 \times$ SSC, two 20 minute washes in $2 \times$ SSC, and two 20 minute washes in $0.1 \times$ SSC at $42^{\circ} \mathrm{C}$ or $37^{\circ} \mathrm{C}$. A final wash in $2 \times$ SSC at room temperature preceded washing in alkaline phosphatase buffer, $\mathrm{pH} 9 \cdot 5$. The slides were incubated in nitroblue tetrazolium (NBT)/5-bromo-4-chloro-3-indolyl phosphate (BCIP) for two hours. Levamisole was included to block endogenous alkaline phosphatase. Slides were then counterstained with haematoxylin and mounted using Glycergel (Dako).

Controls included incubation of sections with unlabelled probe, and pretreatment of sections with RNAse A, as previously described. $^{4}$

Total RNA was extracted from rat pituitaries using RNAzol (Biogenesis Ltd), run out on a denaturing agarose gel and transferred to a nitrocellulose filter. Lanes containing $7 \mu \mathrm{g}$ and $4 \mu \mathrm{g}$ RNA were run in duplicate. One half of

Figure 1 In situ hybridisation of rat $m R N A$ with directly linked to alkaline phosphatase. (A) $0 \%$ formamide buffer; (B)

$50 \%$ formamide buffer. In the absence of formamide a strong signal is seen in the intermediate lobe (arrows) and in a scattered population of stellate cells in the anterior lobe. The inclusion of formamide reduces the signal in the intermediate lobe and abolishes the signal in the anterior lobe. the filter was probed with alkaline phosphatase POMC probe in $50 \%$ formamide hybridisation buffer while the other half was probed using $0 \%$ formamide hybridisation buffer.

Hybridisation was allowed to proceed overnight at $50^{\circ} \mathrm{C}$. The post-hybridisation washes were carried out at $50^{\circ} \mathrm{C}$ and comprised $1 \times \mathrm{SSC} / 0.5 \%$ sodium dodecyl sulphate (SDS) twice, followed by two washes of $0.25 \times \mathrm{SSC} / 0.5 \%$ SDS. The blots were washed once in $2 \times$ SSC to remove all traces of SDS, then washed in alkaline phosphatase buffer, $\mathrm{pH} 9 \cdot 5$. The blots were then incubated in NBT/BCIP substrate solution until bands were visible.

Cryostat sections ( $5 \mu \mathrm{m})$ were cut from rat kidneys and mounted on silanised slides, as with fixed tissues for in situ hybridisation. These were air dried for one hour and then fixed in acetone for five minutes. Paired sections were washed in water and incubated in either $50 \%$ formamide or $0 \%$ formamide hybridisation buffer overnight at $42^{\circ} \mathrm{C}$ under siliconised coverslips. The sections were then rinsed in $4 \times$ SSC to remove coverslips and washed in alkaline phosphatase buffer, $\mathrm{pH} 9 \cdot 5$. Sections were incubated in NBT/BCIP for one hour at room temperature, washed in water, lightly counterstained with haematoxylin and mounted in Glycergel (Dako).

Using an indirect immunoperoxidase technique, sections of rat pituitary were immunostained using a primary antibody raised in rabbit to adrenocorticotrophin (ACTH) (Dako). Sections of pancreas were stained using a primary antibody raised in rabbit to somatostatin (Dako). Secondary antibodies were goat anti-rabbit (Dako).

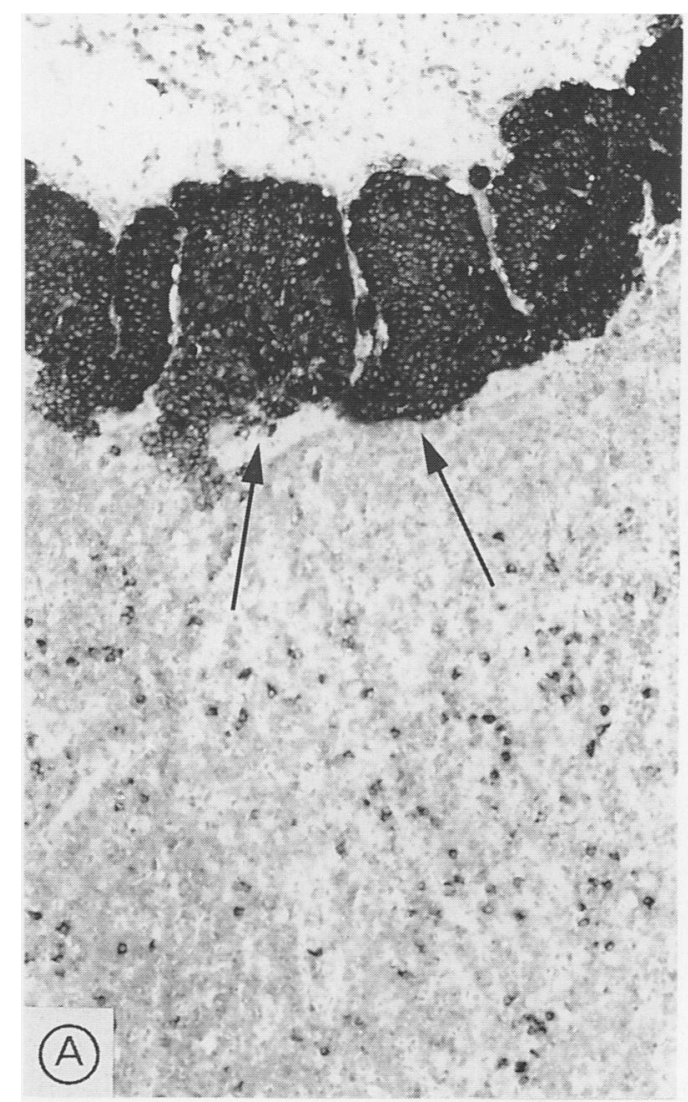


Sites of binding were visualised using 3'3' diaminobenzidine as chromogen.

\section{Results}

IN SITU HYBRIDISATION

In $0 \%$ formamide buffer a strong signal for POMC mRNA was seen in the intermediate lobe and in a scattered population of stellate cells in the anterior lobe of the rat pituitary (fig 1A). In the human pancreas scattered individual cells were positive in the islets of Langerhans (fig 2A). The subjective intensity of signal was similar to that obtained using digoxigenin in the presence of formamide, as described previously. ${ }^{4}$ The distribution of the signals was similar to that of the gene product detected by immunohistochemistry (data not shown). Incubation in $50 \%$ formamide hybridisation buffer significantly decreased the signal for POMC MRNA in the intermediate lobe and almost abolished it in the anterior lobe (fig 1B). The signal for somatostatin mRNA in the islets was abolished (fig 2B). Signal was abolished by pretreatment with RNAse. No staining was seen when sections were incubated with unlabelled probe.

\section{NORTHERN BLOT ANALYSIS}

In both $0 \%$ formamide buffer (fig 3 ) and in $50 \%$ formamide buffer, the POMC probe detected a single band of appropriate size (about 1200 base pairs). However, the signal was more intense in the $0 \%$ formamide buffer, and developed within two hours; the weak signal in $50 \%$ formamide took five days of incubation to become apparent.

Figure 2 In situ hybridisation of human pancreas for somatostatin $m R N A$ with oligoprobe directly linked to alkaline phosphatase. $(A) 0 \%$ formamide buffer; $(B)$ $50 \%$ formamide. In the absence of formamide signal is seen in a subpopulation of islet cells (arrows). It is abolished by formamide.

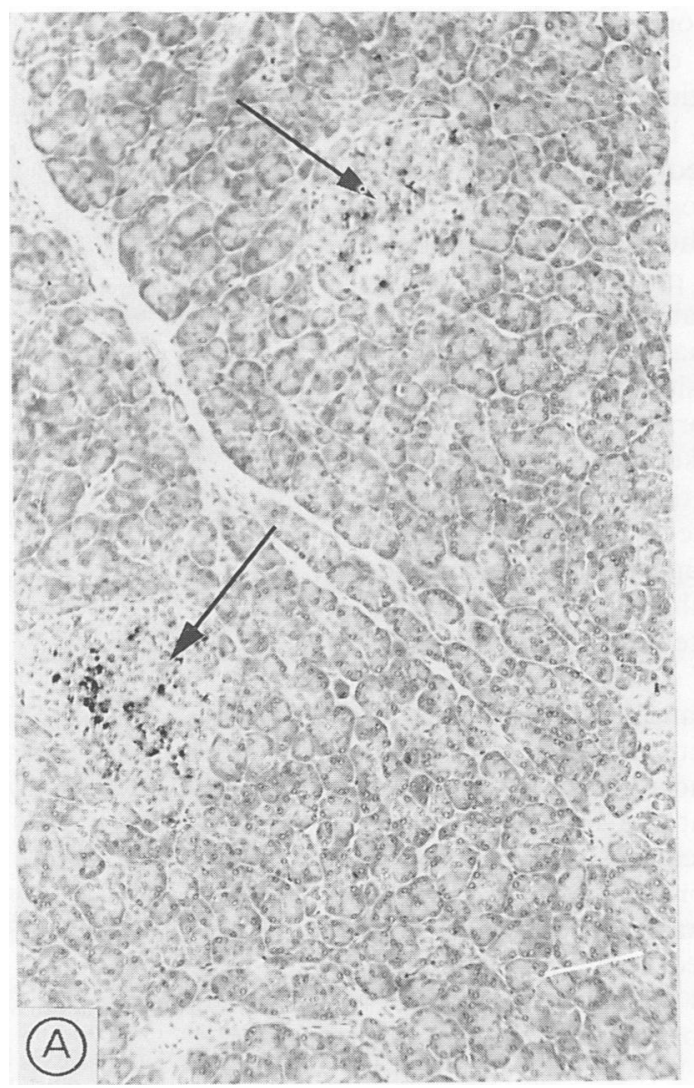

HISTOCHEMICAL DETECTION OF ALKALINE PHOSPHATASE

Endogenous alkaline phosphatase activity was clearly visible in rat kidney after preincubation in $0 \%$ formamide buffer (fig $4 \mathrm{~A}$ ). Preincubation in $50 \%$ formamide buffer abolished the signal (fig 4B).

\section{Discussion}

Hybridisation procedures using oligodeoxynucleotide probes have been adapted directly from methods used initially with cDNA probes or riboprobes. ${ }^{8-10}$ With such long sequences mismatched non-specific hybrids may be formed which may be dissociated by washing at high temperature. This has the disadvantage of destroying tissue architecture. Alternatively, the inclusion of formamide in the mixture reduces the melting point of hybrids, thus permitting stringent washing of tissue sections at temperatures which will not damage the structure. The application of this technique using synthetic oligoprobes labelled with radioisotopes, ${ }^{11} 12$ biotin, ${ }^{1-3}$ and digoxigenin ${ }^{45}$ has permitted the localisation of specific mRNAs in tissue sections and in blot analysis. ${ }^{45}$ However, with the short sequences used in oligomer probes, the likelihood of formation of nonspecific hybrids is decreased and therefore the requirement for such stringent washing may be reduced.

In the present study using the POMC probe, we have shown that specific mRNA can be detected in northern blot analysis using an oligoprobe directly linked to alkaline phosphatase. We have also shown that strong specific signal, without background, can be identified

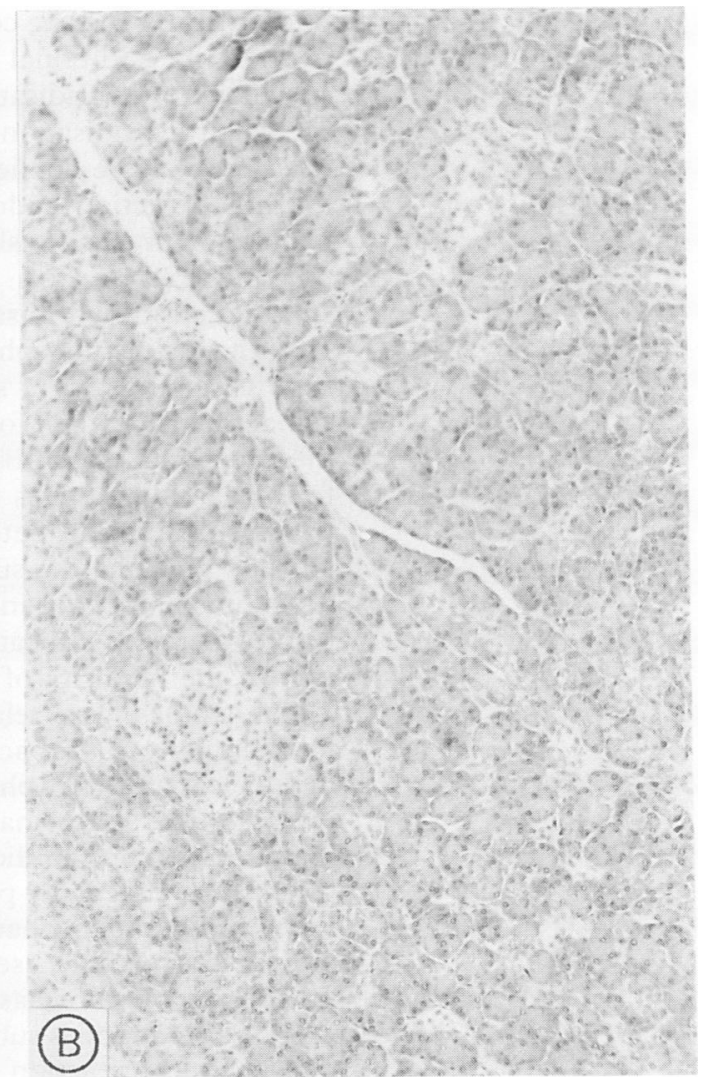




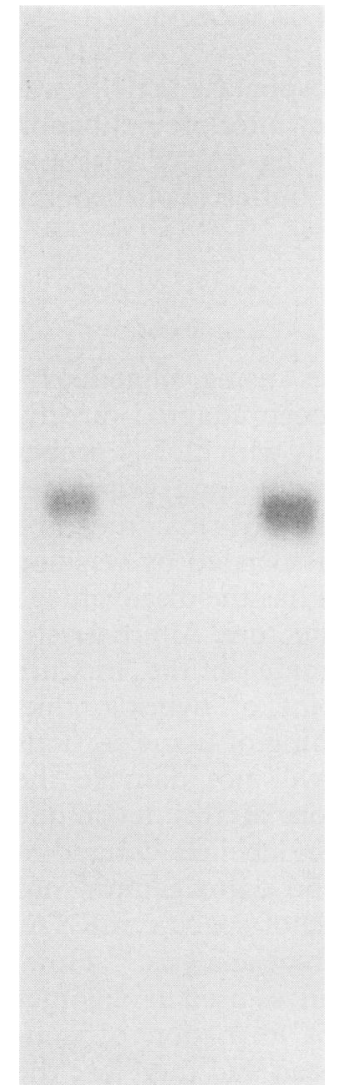

Figure 3 Northern blot analysis for POMC mRNA using an oligoprobe directly linked to alkaline phosphatase, in $0 \%$ formamide buffer. The probe detects a single band of appropriate size.
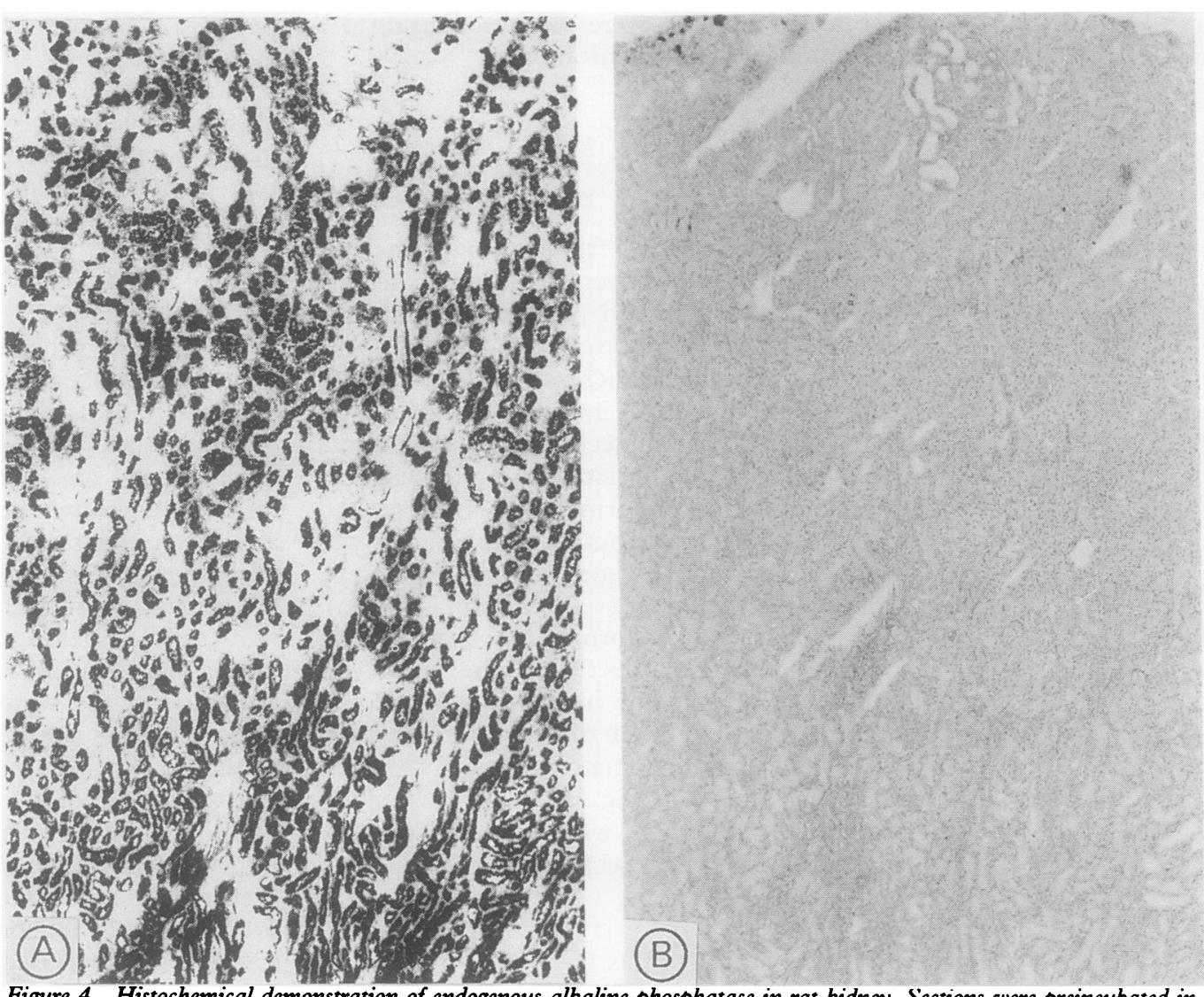

Figure 4 Histochemical demonstration of endogenous alkaline phosphatase in rat kidney. Sections were preincubated in (A) $0 \%$ formamide buffer and (B) 50\% formamide buffer. Preincubation in formamide abolishes the reaction.

in the absence of formamide, and that there are clear signals for both POMC and somatostatin $\mathrm{mRNAs}$ with in situ hybridisation, again without background. The specificity of POMC probe binding has already been reported ${ }^{4}$; the concordance between the staining patterns for both $\mathrm{mRNAs}$ and the corresponding hormonal peptides is additional evidence in favour of specificity of hybridisation. ${ }^{5}$ The lack of staining when hybridisation buffer alone or unlabelled probe were applied also indicate that we are not detecting endogenous alkaline phosphatase. Our results show that formamide is not required in this procedure to provide specific, clean hybridisation.

With alkaline phosphatase linked probes, we have indeed shown a significant reduction in signal intensity when formamide is included in the procedure. Our observation that exposure to formamide reduces the signal generated in the histochemical detection of endogenous alkaline phosphatase suggests that formamide directly denatures or inhibits the enzyme. Our results therefore indicate that the major reduction in the intensity of signal observed in the initial studies was related to the effects of formamide on the activity of the reporter molecule, alkaline phosphatase. We would propose, therefore, that the inclusion of formamide is contraindicated in hybridisation techniques based on probes directly labelled with alkaline phosphatase. Interestingly, the dimethylformamide used in the histochemical incubation mixture has no such effect. However, if formamide is substituted at this step the histochemical reaction is inhibited (data not shown). In view of the known toxic effects of the chemical the ability to omit formamide from procedures based on non-isotopic labelling of oligoprobes enhances their safety and general applicability in diagnostic practice.

We thank Drs J Parker and A Garven of Cambridge Research Biochemicals for the gift of the probes and Mrs Joan Cramb for typing the manuscript.

1 Guitteny A, Fouque B, Mougan C, Teoule R, Bloch B. Histological detection of messenger RNAs with biotinylated synthetic oligonucleotide probes. $F$ Histochem Cytochem 1988;36:563-71.

2 Pringle JH, Homer CE, Warford A, Kendall $\mathrm{CH}$, Lauder I. In situ hybridization: alkaline phosphatase visualization of biotinylated probes in cryostat and paraffin sections. Histochem $\mathcal{F}$ 1987;19:488-96.

3 Brigati DJ, Myerson D, Leary JJ, et al. Detection of viral genomes in cultured cells and paraffin-embedded tissue sections using biotin labelled hybridization probes. Virology 1983;126:32-50.

4 Farquharson M, Harvie R, McNicol AM. Detection of messenger RNA using a digoxigenin end labelled oligodeoxynucleotide probe. $\mathcal{f}$ Clin Pathol 1990;43:424-8.

5 Shorrock K, Roberts P, Pringle JH, Lauder I. Demonstration of insulin and glucagon mRNA in routinely fixed and processed pancreatic tissue by in situ hybridization. $\mathcal{F}$ Pathol 1991;165:105-10.

6 Larsson LI, Christensen T, Dalboge H. Detection of proopiomelanocortin mRNA by in situ hybridization, using a biotinylated oligodeoxynucleotide probe and avidin-alkaline phosphatase histochemistry. Histochemistry 1988;89:109-16.

7 Montminy MR, Goodman RH, Horovitch SJ, Habener JF. Primary structure of the gene encoding rat preproso-
matostatin. Proc Natl Acad Sci USA 1984;81:3337-40.

8 Brahic M, Haase AT. Detection of viral sequences of low reiteration frequency by in situ hybridization. Proc Natl reiteration frequency by in situ hy
Acad Sci USA 1978;75:6125-9.

9 Gee C, Roberts JL. In situ hybridization histochemistry: A technique for the study of gene expression in single cells. DNA 1983;2:157-63.

10 Cox KH, Deleon DV, Angerer LM, Angerer RC. Detection of mRNAs in sea urchin embryos by in situ hybridization using asymmetric RNA probes. Dev Biol 1984;101: 485-502

11 Taneja K, Singer RH. Use of oligodeoxynucleotide probes for quantitative in situ hybridization to actin mRNA. Anal Biochem 1987;166:389-98.

12 Priestley JV, Hynes MA, Han VKM, Rethelyi M, Perl ER, Lundl PK. In situ hybridization using ${ }^{32} \mathrm{P}$ labelled oligodeoxy-ribonucleotides for the cellular localisation of mRNA in neuronal and endocrine tissue. Histochemistry 1988;89:467-79. 